\title{
Inflammatory status and bone phenotype: results from the National Survey of Health and Development
}

\author{
R. Durdin ${ }^{1}$, C. Parsons ${ }^{1}$, D. Kuh ${ }^{2}$, E.M. Dennison ${ }^{1}$, R. Cooper $^{3}$, C. Cooper $^{1}$ and K.A. Ward ${ }^{1}$ \\ ${ }^{1}$ MRC Lifecourse Epidemiology Unit, University of Southampton, Southampton General Hospital, Southampton, UK, \\ ${ }^{2}$ MRC Unit for Lifelong Health and Ageing, University College London, London, UK and \\ ${ }^{3}$ Manchester Metropolitan University, Manchester, UK
}

Fragility fractures associated with osteoporosis cause significant morbidity and mortality; it is therefore important to understand the determinants of poorer bone health in older age to maintain quality of life. Ageing is associated with a low-grade level of inflammation, which is suggested as a risk factor for several chronic conditions including bone health, and adipose tissue is a source of inflammatory mediators including the adipokines, adiponectin and leptin ${ }^{(1)}$. While links between inflammatory mediators and bone health have been described ${ }^{(2)}$ few studies have investigated relationships with bone microarchitecture ${ }^{(3)}$. The aim was to investigate the associations between interleukin-6 (IL-6), adiponectin and leptin with bone phenotype measured via both dual energy X-ray absorptiometry (DXA) and peripheral quantitative computed tomography (pQCT).

The National Survey of Health and Development (NSHD) is a prospective birth cohort study; 766 men and 820 women aged 60-64 years were included in these cross-sectional analyses. Participants had DXA (total hip, spine, whole body areal BMD (aBMD)) and pQCT (radius: 4\% and 50\% sites) measurements. Multiple linear regression, stratified by sex, was used to determine associations between inflammatory markers (IL-6, adiponectin and leptin) and bone, with adjustment for fat mass residuals (the residual of fat mass after adjustment for lean mass and height). Results are presented as $\beta(95 \%$ confidence interval) $\mathrm{p}$-value; $\beta$ is the change in aBMD $(\mathrm{g} / \mathrm{cm} 2)$ or volumetric BMD (vBMD, $\mathrm{mg} / \mathrm{mm} 3)$ per unit increase in inflammatory marker.

In women, positive associations between $\log (\mathrm{IL}-6)$ and aBMD did not remain after adjustment for fat mass residuals (hip: 0.004 $(-0.009,0.02) 0.56)$, whereas associations with total vBMD did remain after adjustment $(10.30 \mathrm{mg} / \mathrm{mm} 3(2.01,18.58) 0.02))$. In $\mathrm{men}$, negative associations existed between $\log (\mathrm{IL}-6)$ and hip aBMD (-0.02g/cm2 (-0.03,-0.003) 0.02) and total vBMD (-8.48mg/mm3 $(-16.24,-0.72) 0.03)$. Log(IL-6) was not associated with medullary or cross-sectional area in men or women. In men and women, $\log ($ leptin) was positively associated with hip aBMD (men: $(0.02 \mathrm{~g} / \mathrm{cm} 2(0.002,0.04) 0.03)$; women: $(0.03 \mathrm{~g} / \mathrm{cm} 2(0.02,0.05)<0.001))$ and trabecular vBMD (men: $(7.69 \mathrm{mg} / \mathrm{mm} 3$ (1.72,13.66) 0.01$)$; women: $(8.73 \mathrm{mg} / \mathrm{mm} 3(2.99,14.47)$ 0.003)); after adjustment, positive associations with cortical vBMD existed in women only. In men and women, adiponectin was negatively associated with aBMD, at all sites, and radius trabecular vBMD. Associations between adiponectin and both cortical and total vBMD existed in women only (total vBMD: $-1.43 \mathrm{mg} / \mathrm{mm} 3(-2.00,-0.85)<0.001)$.

Associations between IL-6, adiponectin and leptin with bone were found. There were differences in the direction of association of IL-6 and total vBMD between men and women, and in both men and women, we found positive associations between leptin and DXA-based measures of bone density, and negative associations between adiponectin and bone density. These results contribute to our understanding of the relationships between inflammatory status and bone.

\section{References}

1. Ferrucci L \& Fabbri E (2018) Nat Rev Cardiol 15(9), 505-22.

2. Cauley JA, Danielson ME, Boudreau RM, et al. (2007) J Bone Miner Res 22(7), 1088-95.

3. Rolland T, Boutroy S, Vilayphiou N, et al. (2012) Calcif Tissue Int 90(6), 496-506. 